- RAM, REV. ADM. MACKENZIE, V. 12, N. 3, Edição Especial • SÃO PAULO, SP • MAIO/JUN. 2011 • ISSN 1678-6971 • Submissão: 16 jan. 2011. Aceitação: 18 mar. 2011. Sistema de avaliação: às cegas dupla (double blind review). UNIVERSIDADE PRESBITERIANA MACKENZIE. Walter Bataglia (Ed.), p. 21-50.

\title{
EDUCAÇÃO PARA A SUSTENTABILIDADE NOS CURSOS DE ADMINISTRAÇÃO: REFLEXÃO SOBRE PARADIGMAS E PRÁTICAS
}

PEDRO ROBERTO JACOBI

Doutor em Sociologia pela Faculdade de Filosofia, Letras e

Ciências Humanas da Universidade de São Paulo (USP).

Professor da Faculdade de Educação e do Programa de Pós-Graduação em Ciência Ambiental da Universidade de São Paulo.

Avenida Luciano Gualberto, 1.289, Procam-USP - IEE, Prédio da Divisão de Ensino e Pesquisa, $2^{\circ}$ andar, sala S16, Secretaria de Pós-Graduação, Cidade Universitária, São Paulo - SP - Brasil - CEP 05508-010

E-mail: priacobi@usp.br

EMMANUELL RAUFFL트

PhD em Gestão pela Faculdade de Gestão da Universidade de McGill. Professor do Departamento de Gestão da Escola de Negócios Internacional HEC Montréal. 3000, chemin de la côte Ste Catherine, Montreal - Quebec - Canadá - H3AT2A7

E-mail: emmanuel.raufflet.hec.ca

MICHELLEE PADOVESE DE ARRUDA

Mestranda em Ciência Ambiental pela Universidade de São Paulo (USP). Avenida Luciano Gualberto, 1.289, Procam-USP - IEE, Prédio da Divisão de Ensino e Pesquisa, $2^{\circ}$ andar, sala S16, Secretaria de Pós-Graduação, Cidade Universitária, São Paulo - SP - Brasil - CEP 05508-010

E-mail: michellearruda@usp.br 


\section{RESUMO}

Na última década, multiplicaram-se os módulos, cursos e programas relacionados à sustentabilidade no ensino superior em geral, assim como mais especificamente no ensino da Administração. O propósito deste artigo é apresentar uma reflexão e uma avaliação de tais esforços e mapear caminhos para a construção de módulos, cursos e programas por meio de uma reflexão sobre paradigmas da educação e suas práticas. O objetivo é contribuir no debate sobre o papel da educação superior e notadamente dos cursos de Administração e Gestão, na medida em que se observa um significativo aumento do número de instituições de ensino superior que apresentam efetiva motivação para formar estudantes com conhecimento e habilidades necessários para colocar a sustentabilidade no centro de suas futuras atividades de gestão. Apresenta-se uma reflexão sobre as principais vertentes de pensamento e os debates atuais, identificam-se as ambiguidades e contradições, e se apresentam as possibilidades de integração do tema da sustentabilidade nos cursos de Administração. Os autores trazem à tona a temática da sustentabilidade nas empresas e seu diálogo com a academia, bem como as implicações da emergência da sociedade de risco para a educação superior e as escolas de Administração. Também se abordam as correntes de pensamento da economia e ecologia propostas para a educação, dentro de paradigmas que tratem da complexidade e inserção da sustentabilidade na educação superior e nas escolas de Administração. O artigo está estruturado em quatro seções: a primeira mapeia a sociedade de risco e o pensamento econômico recente, e esboça os fundamentos/premissas da sociedade, bem como os paradigmas da educação; a segunda apresenta um levantamento sobre promoção/difusão da sustentabilidade na educação superior, destacando contexto, resultados e desafios; a terceira foca a sustentabilidade no ensino da Administração e seus principais desafios; e a última seção propõe três caminhos para integrar o tema da sustentabilidade no ensino da Administração, com as competências para o desenvolvimento sustentável, e, 
finalmente, as implicações, os obstáculos e as possibilidades de integrar a sustentabilidade no ensino da Administração. O artigo dialoga com a literatura internacional mais recente e busca interessar os leitores sobre os principais desafios conceituais para educar indivíduos responsáveis e comprometidos com a sustentabilidade.

\section{PALAVRAS-CHAVE}

Sustentabilidade; Ensino superior; Ensino de Administração; Paradigmas educativos; Sociedade de risco.

\section{INTRODUÇÃO: A TEMÁTICA DA SUSTENTABBILIDADE SOCIOAMBIENTAL NAS EMPRESAS E SEU DIÁLOGO COM A ACADEMIA}

Durante a última década, muitos programas de graduação, pós-graduação e especialização em negócios têm inserido módulos, cursos e componentes de sustentabilidade em seus currículos. Vários programas relacionados à sustentabilidade também foram criados (STARIK; RANDS, 2009, para uma visão geral sobre os Estados Unidos). Publicaram-se volumes e edições especiais de jornais sobre tais experiências e lições aprendidas (WANKEL; STONER, 2008, 2009; BUSINESS STRATEGY AND THE ENVIRONMENT, 2005; SLATER; DIXON-FOWLER, 20IO), e muitos fóruns foram estabelecidos para que educadores de gestão e negócios pudessem trocar e discutir experiências e, de forma mais ampla, refletir sobre tópicos relacionados às questões da sustentabilidade no ensino da Administração.

Neste artigo, define-se sustentabilidade como a capacidade de resistir, durar. $\mathrm{Na}$ ecologia, a palavra sustentabilidade descreve como sistemas biológicos se mantêm diversos e produtivos ao longo do tempo. Florestas e áreas de pantanal longevas são exemplos de sistemas biológicos sustentáveis. Para os indivíduos, a sustentabilidade é o potencial de manutenção de bem-estar por um longo período, o que possui dimensões ambientais, econômicas e sociais.

O objetivo deste artigo é contribuir para o debate sobre o papel da educação superior e notadamente dos cursos de Administração e Gestão, na medida em que se observa um significativo aumento do número de instituições de ensino superior que apresentam uma efetiva motivação para formar estudantes com co- 
nhecimento e habilidades necessários para colocar a sustentabilidade no centro de suas futuras atividades de gestão.

Apresenta-se uma reflexão sobre as principais vertentes de pensamento, os principais debates, identificando ambiguidades e contradições no debate e apresentando as possibilidades da integração do tema da sustentabilidade nos cursos de Administração.

Este artigo está estruturado em quatro partes: a primeira mapeia a sociedade de risco e o pensamento econômico recente, e esboça os fundamentos/ premissas da sociedade, bem como os paradigmas da educação; a segunda parte mapeia a promoção/difusão da sustentabilidade na educação superior, considerando contexto, resultados e desafios; a terceira foca a sustentabilidade no ensino da Administração e seus principais desafios; e a última e conclusiva parte propõe três caminhos para a integração da sustentabilidade no ensino da Administração.

\section{PARTE 1: A EMERGÊNCIA DA SOCIEDADE DE RISCO}

O ponto de partida sobre a definição de sociedade fundamenta-se na noção de Ulrich Beck (I992) sobre sociedade de risco. A multiplicação dos riscos, em especial os ambientais e tecnológicos de graves consequências, é o elementochave para entender as características, os limites e as transformações de nossa modernidade.

A sociedade, produtora de riscos, torna-se cada vez mais reflexiva, mais autocrítica, e o conceito de risco passa a ocupar papel estratégico para entender as características, os limites e as transformações do projeto histórico da modernidade (BECK; GRANDE, 20IO).

A emergência da "sociedade de risco" e das respostas a esses riscos, convertidas em pactos planetários, em programas direcionados a mudanças nos procedimentos produtivos e nas ações educativas em escala global, é ilustrada pela Figura I. A reflexão sobre "sociedade de risco" permite abordar a complexa temática das relações entre sociedade, meio ambiente e educação. 


\section{FIgURA I}

PRINCIPAIS DESASTRES AMBIENTAIS I920-20I0

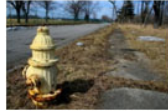

Contaminação em Love Canal $-1920$

Minamata, Japão - 19281968
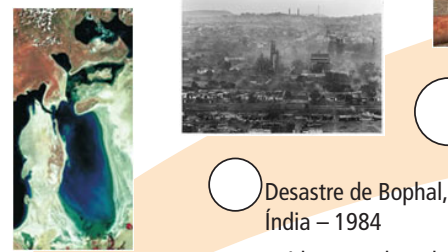
Índia - 1984

Acidente nuclear de Chernobyl, Ucrânia $-1986$ Mar Aral - 60's Província de Henan, China 1975 Meda, Itália - 197

Exxon Valdez, Alasca - 1989

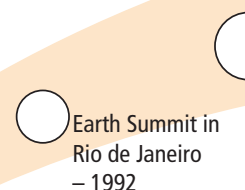
$-1992$

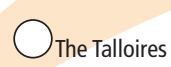
Declaration -1990 University Leaders for a Sustainable

Relatório Brundtland 1987

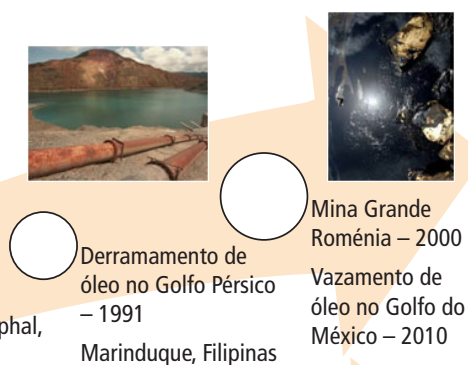

- 1996

Tokai, Japão - 1999

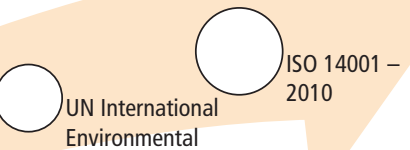

Education Program

Education Program

$-2004$

The United Nations Decade of Education for Sustainable Development 2005-2014

Fonte: Elaborada pelos autores.

De um lado, há os eventos que provocaram mudanças de comportamento e práticas sociais e empresariais; de outro, uma série de iniciativas globais para enfrentar a problemática ambiental, bem como a realização de eventos e encontros que estabeleceram um diálogo do campo acadêmico com a sustentabilidade, do governo com a educação para o desenvolvimento sustentável e a aprovação de inicitiavas do setor empresarial em diálogo com a sociedade civil, a fim de avançar na legitimação de instrumentos de certificação que incluem a temática ambiental na produção de bens e mercadorias.

Um bom exemplo disso é a criação da ISO I400I, uma norma que define o que deve ser feito para estabelecer um sistema de gestão ambiental efetivo, visando identificar, priorizar e gerenciar seus riscos ambientais como parte de suas práticas usuais. 


\subsection{IMPLICAÇÕES DA EMERGÊNCIA DA SOCIEDADE DE RISCO PARA A EDUCAÇÃO SUPERIOR E AS ESCOLAS DE ADMINISTRAÇÃO}

A intensificação e a multiplicação de crises ambientais causadas no contexto da sociedade de risco, desde meados dos anos I990, estimularam as instituições acadêmicas a desenvolver maior compromisso do tema sustentabilidade no ensino superior. Observa-se que os diferentes momentos de eventos emblemáticos no plano ambiental, marcados por acontecimentos que impactaram o meio ambiente e os ecossistemas, envolvem um conjunto de atores do universo educativo, em todos os níveis, potencializando o engajamento dos diversos sistemas de conhecimento e a sua capacitação em uma perspectiva interdisciplinar com ênfase na sustentabilidade.

Os educadores têm desempenhado um papel estratégico e decisivo na inserção da educação para a sustentabilidade, qualificando os alunos para um posicionamento crítico ante a crise socioambiental, tendo como horizonte a transformação de hábitos e práticas sociais e a formação de uma cidadania ambiental que os mobilize para a questão da sustentabilidade em seu significado mais abrangente (JACOBI, 2005).

\section{VISÕES SOBRE ECONOMIA E ECOLOGIA: TRẾS CORRENTES DE PENSAMENTO}

Dado que o pensamento econômico prevaleceu no currículo do ensino da Administração (GHOSHAL, 2005), revisam-se, neste artigo, três paradigmas do pensamento econômico que se referem à relação entre a economia e o ambiente natural. Duas correntes de pensamento prevalecem, a denominada convencional, segundo a qual o crescimento econômico é o determinante para alcançar sustentabilidade; e a economia ecológica, que advoga sobre a economia estacionária.

A posição ecológica é majoritariamente considerada pouco factível, dado que a lógica prevalecente na economia global estimula o consumo e o uso intensivo dos recursos naturais, e a convencional é inconsistente com as grandes questões ambientais globais (VEIGA, 20IO). Os economistas ecológicos olham para as limitações globais, seja pela ótica dos recursos e serviços naturais, sua finitude ou rapidez de dilapidação, seja pela capacidade - ou incapacidade - de absorção de resíduos.

As discussões sobre sustentabilidade nas escolas de Administração possuíam, em seu início, um foco majoriariamente econômico. A leitura designa- 
da aos estudantes era principalmente de clássicos, como The tragedy of the commons, de Garrett Hardin (I968), e Steady-state economics, de Herman Daly (I977). Durante vinte anos, o curso de Administração se manteve fortemente enraizado no conceito da economia como um sistema aberto. No entanto, o curso evoluiu de centrado em conceitos econômicos para um enfoque estratégico-administrativo (STEAD; STEAD, 2010).

Outra vertente de economistas aposta na necessária reconfiguração do processo produtivo, com crescentes ganhos em ecoeficiência, com uso cada vez menos intensivo de energia, e isso permitiria que a economia continuasse a crescer, sem que limites ecológicos fossem rompidos ou pelo esgotamento dos recursos naturais (VEIGA, 20IO). Entretanto, essa vertente também tem sido objeto de questionamentos, tendo em Tim Jackson (2009), autor do relatório Prosperity without growth? Economics for a finite Planet, o debate do dilema do crescimento exigindo o rompimento mental com uma macroeconomia inteiramente centrada no ininterrupto aumento do consumo, em vez de uma visão keynesiana pautada pela ecoeficiência (VEIGA, 20IO).

Jackson (2009) defende que o descolamento entre crescimento e emissões de gases-estufa não acontecerá na velocidade necessária para impedir danos catastróficos à segurança climática, o que o leva a propor a adoção de um novo paradigma pós-crescimento.

Atualmente, o avanço rumo a uma sociedade assentada em valores de sustentabilidade, rumo à descarbonização, é muito lento ante as perspectivas colocadas para se contrapor ao aquecimento global. No entanto, novas propostas conceituais que nos instigam à reflexão começam a surgir, tal como o conceito da econologia ${ }^{\mathrm{I}}$ ou o que se define como a junção entre a economia e a ecologia.

\section{PROPOSTAS PARA EDUCAÇÃO: EM DIREĈ̃O A PARADIGMAS QUE ABORDEM A COMPLEXIDADE}

A sustentabilidade como novo critério básico e integrador pode fortalecer valores coletivos e solidários a partir de práticas educativas contextualizadoras e problematizadoras, que, pautadas pelo paradigma da complexidade, aportem para a escola e para outros ambientes pedagógicos uma atitude de ação-reflexão-ação em torno da problemática ambiental. 
As práticas educativas ambientalmente sustentáveis apontam para propostas pedagógicas centradas na criticidade dos sujeitos, com vistas à mudança de comportamento e atitudes, ao desenvolvimento da organização social e da participação coletiva. Essa mudança paradigmática implica uma mudança de percepção e de valores, gerando um pensamento complexo, aberto às indeterminações, às mudanças, à diversidade, à possibilidade de construir e reconstruir, em um processo contínuo de novas leituras e interpretações, configurando novas possibilidades de ação.

A premissa que norteia o paradigma proposto é o diálogo de saberes que permita construir espaços de fronteiras; formar um pensamento crítico, criativo e sintonizado com a necessidade de propor respostas para o futuro, capaz de analisar as complexas relações entre os processos naturais e sociais e de atuar no ambiente em uma perspectiva global, respeitando as diversidades socioculturais (JACOBI, 2005).

As experiências e práticas educativas e de pesquisa interdisciplinares ainda são recentes e incipientes. Os processos de conhecimento buscam estabelecer cortes transversais na compreensão e explicação dos contextos de aprendizagem e de formação. O estímulo é para a interação e interdependência entre as disciplinas e, consequentemente, entre as pessoas para o desenvolvimento de metodologias interativas.

\section{PARTE 2: EDUCAÇÃO SUPERIOR E INSERÇÃO DO ŢEMA SUSTENT'ABILIDADE}

As instituições de ensino superior não estão apenas educando as futuras gerações para tomadores de decisão, tais instituições têm papel importante na trajetória para um futuro global mais sustentável. Tal papel é exercido por meio de três componentes: I. os espaços de formação, intercâmbio e educação; 2 . os espaços de pesquisa e geração de ideias; e 3 as organizações per se, com orçamentos e processos de tomada de decisão.

\subsection{ESPAÇOS EDUCATIVOS EM TRANSFORMAÇÃO}

Deve-se recordar que o conceito de sustentabilidade no ensino superior não é novo e remonta aos diálogos internacionais que antecederam e que ocorreram durante a Earth Summit, no Rio de Janeiro, em I992, e que o UN International Environmental Education Program foi o primeiro a introduzir a noção de sustentabilidade no ensino superior (TILBURY, 2004). 
Tem havido iniciativas tanto no nível internacional quanto no nacional para promover a sustentabilidade nas instituições de ensino superior, desde os anos I990.

A Década das Nações Unidas da Educação para o Desenvolvimento Sustentável 2005-20I4 encontra-se em estágio bastante avançado, e países ao redor do mundo estão tomando medidas progressivas para integrar a sustentabilidade nos planos de educação, em todos os níveis e em todos os setores, incluindo o ensino superior. Uma das questões principais é incluir a educação para a sustentabilidade no currículo de ensino superior, e, entre os maiores desafios, está o de expandir a sustentabilidade nos sistemas educativos.

De acordo com Tilbury e Wortman (2008), há um número crescente de instituições de ensino superior em vários países que está encontrando apoio, em nível nacional, para fortalecer uma formação pró-sustentabilidade. No caso do Reino Unido, o Sustainable Development Education Panel and Higher Education Partnership for Sustainability fez recomendações ao governo para mudanças em muitos setores, incluindo o ensino superior, a fim de treinar plenamente todos os altos membros do ensino superior do Reino Unido em sustentabilidade, bem como proporcionar aos alunos oportunidades de aprendizagem relevantes para a sustentabilidade.

Os governos nacionais da Austrália ${ }^{2}$, Nova Zelândia ${ }^{3}$ e África do Sul também reconhecem a importância de maior envolvimento em sustentabilidade e de aprendizagem ambiental no ensino superior.

\subsection{A MULTIPLICAÇÃO DE INICIATIVAS NAS INSTITUIÇÕES DE ENSINO SUPERIOR}

Durante a década de I990, um número crescente de instituições de ensino superior se comprometeu institucionalmente com a sustentabilidade, com a assinatura de declarações de sustentabilidade nacional e internacional (ASSOCIATION OF UNIVERSITY LEADER FOR A SUSTAINABLE FUTURE, I990), e tais declarações se tornaram quadros influentes na difusão de princípios de sustentabilidade e práticas em instituições de ensino superior (WRIGHT, 2002, 2004).

A Declaração de Talloires, composta em I990, em uma conferência internacional na França, é a primeira declaração oficial, feita por gestores universitários,

Para saber mais sobre iniciativas governamentais em sustentabilidade, ver o site do The Australian Research Institute for Environment and Sustainability (Aries): http://www.aries.mq.edu.au/.

3 Para parcerias entre escolas de Administração e corporações para a sustentabilidade, ver o site do New Zealand Business Council for Sustainable Development: http://www.nzbcsd.org.nz/schools.asp. 
de um compromisso com a sustentabilidade ambiental no ensino superior, bem como a declaração de sustentabilidade com mais amplo apoio internacional até a presente data.

A Declaração de Talloires (TD) é um plano de ação de dez pontos para incorporar a sustentabilidade e a instrução ambiental no ensino, na pesquisa e na extensão de operações em faculdades e universidades. Foi assinada por mais de 350 presidentes e reitores de universidades de mais de 40 países. É importante porque reconhece que a mudança curricular é necessária em todos os programas de estudo, e não apenas para aqueles com foco na sustentabilidade (ASSOCIATION OF UNIVERSITY LEADER FOR A SUSTAINABLE FUTURE, I990), e que uma universidade sustentável deve integrar a sustentabilidade em todo o currículo, focar a sustentabilidade em pesquisas, contatar outros setores da sociedade, criar uma gestão de campus e operações sustentável, e oferecer oportunidades para o envolvimento dos estudantes.

Entre tais iniciativas, destacam-se os Principles for Responsible Management Education (PRME) no campo da Administração de Empresas, que, até dezembro de 20Io, já tinham sido assinados por mais de 300 instituições de ensino superior de Administração em todo o mundo (ZELEM; BLANCHARD; LECOME, 20Io).

Os seis princípios são: I. Propósito: desenvolver as habilidades dos estudantes para que se tornem futuros geradores de valores sustentáveis nos negócios e na sociedade e trabalhar para uma economia global sustentável; 2. Valores: incorporar nas atividades acadêmicas e curriculares os valores globais da responsabilidade social, tal qual definidos por iniciativas internacionais, como a United Nations Global Compact; 3. Método: criar estruturas educacionais, materiais e processos que possibilitem experiências efetivas de aprendizagem para uma liderança responsável; 4. Pesquisa: engajar-se em pesquisas conceituais e empíricas que colaborem para avançar no entendimento sobre o papel, a dinâmica e o impacto das corporações na criação de valores social, ambiental e economicamente sustentáveis; 5. Parceria: interagir com administradores de corporações empresariais para expandir o conhecimento de seus desafios em cumprir suas responsabilidades socioambientais e explorar esforços conjuntos para abordar tais desafios; e 6. Diálogo: facilitar e apoiar diálogos e debates entre educadores, empresas, governo, consumidores, mídia, organizações da sociedade civil e outros grupos de interesse e stakeholders em questões críticas relacionadas à responsabilidade social global e à sustentabilidade. Essas ações e atividades relacionadas com a sustentabilidade nas instituições se manifestaram de duas formas inter-relacionadas.

A primeira delas está relacionada com iniciativas dentro dos campi, que consistem no que se convencionou denominar planos para "campus greening", e o desenvolvimento de programas para promover a reciclagem, eficiência energé- 
tica, conservação de água e outras práticas semelhantes. O desempenho positivo de tais projetos tem incentivado, desde então, iniciativas focadas em áreas como gestão de águas pluviais, conservação de energia, transportes sustentáveis e, mais recentemente, na redução de gases de efeito estufa e de compensação de carbono. Assim, a ênfase se coloca no campus como um laboratório para experimentação, para a vida real e aplicação na solução de problemas.

Huckle e Sterling (I996) apresentam um ponto interessante, quando elaboram abordagens inclusivas, envolvendo funcionários administrativos, professores e estudantes que podem ser muito relevantes para desenvolver um suporte para a mudança. As experiências mostram que planos de gestão ambiental e de sustentabilidade do campus são mais bem-sucedidos quando são fundamentados no envolvimento ativo da maior parte da comunidade de um campus, pois a participação em atividades pode ser educativa e criar uma maneira de comprometer mais profundamente alunos e professores para a sustentabilidade (CALVO; BENAYAS; GUTIERREZ, 2002).

O maior desafio é superar as barreiras prevalentes em instituições de ensino superior, a falta de consenso sobre a sustentabilidade, a falta de interesse e comprometimento, as limitadas recompensas para a inovação, a falta de experiência e apoio financeiro (THOMAS, 2004). Basicamente, isso implica o que Tilbury (2004) reconhece como a necessidade de buscar oportunidades, com base em uma visão de futuro compartilhada, oferecendo oportunidades de participação e diálogo, bem como de construir a capacitação para que a sustentabilidade possa se inserir mais profundamente na prática institucional.

A segunda forma tem consistido na transferência para a sustentabilidade no currículo (SHARP, 2002; STERLING, 2004; TILBURY, 2004). Para Raufflet (2006), existem três níveis de aplicação da noção de sustentabilidade nos cursos, programas e atividades curriculares. O primeiro, nos cursos existentes ou nos novos cursos, consiste basicamente em inserir as temáticas ou o material pedagógico em disciplinas existentes. O segundo, no nível de programas, já implica três opções: I. inserção de um ou mais cursos sobre o tema em um programa existente; 2 . inserção de questões centrais da sustentabilidade nos cursos existentes; e 3. reelaboração do programa com base nas questões colocadas pelo enfoque da sustentabilidade, que assim se tornam os campos de aplicação em torno da resolução de problemas e da tomada de consciência individual e coletiva nas quais as disciplinas, os saberes e o conhecimento adquirido são mobilizados e colocados em questão. O terceiro, no nível da reflexão pedagógica e ontológica, consiste em ter uma visão abrangente de sociedade e de formação, e, portanto, contribuir na identificação das implicações quanto aos objetivos e ao conteúdo desses programas e atividades. 


\subsection{OS DESAFIOS PARA PROMOVER A SUSTENTABILIDADE NAS INSTITUIÇÕES DE ENSINO SUPERIOR}

Esses avanços nos níveis de "campus greening" e de currículos sustentáveis vêm enfrentando desafios substanciais.

O principal desafio compreende a ambiguidade e diversidade da definição de sustentabilidade no currículo. A compreensão e a interpretação do termo "sustentabilidade" e a ideia de "aprendizagem para a sustentabilidade" variam muito dentro do ensino superior. A maioria das instituições de ensino superior tem conseguido apenas pequenos avanços incrementais na direção do fortalecimento de uma agenda educacional para a questão da sustentabilidade, confrontadas com a complexidade, bem como com uma incorporada resistência à mudança.

De acordo com Sterling (2004) e Tilbury (2004), mudanças na direção da sustentabilidade no ensino superior requerem mais do que apenas repensar o conteúdo dos currículos de ensino e assinar declarações internacionais, exigindo compromissos com a sustentabilidade em um nível mais profundo, transformando aulas que abordam a sustentabilidade em instituições que trabalham colaborativamente em prol da sustentabilidade. Isso implica uma mudança fundamental não apenas daquilo que se aprende, mas também no contexto e na forma como se aprende. Tais mudanças de conteúdo, contexto e processos educativos exigem um comprometimento mais profundo. Para as instituições acadêmicas e de ensino da Administração, demanda-se um reexame de seus fundamentos, assim como a busca por garantia de coerência entre declarações e homologações, paradigmas educacionais e práticas.

Isso demanda o compromisso institucional de trabalhar para a mudança rumo à sustentabilidade (UNESCO, 2002). Uma agenda institucional, tal como "Aprender para a sustentabilidade", deve envolver o maior número possível de membros da comunidade de uma instituição no cerne de sua cultura, influenciando currículo, formação, financiamento, operações, processos de gestão, ações de pesquisa e outros elementos, como a forma como as instituições de ensino superior ensinam, aprendem e atuam (TILBURY, 2004).

\subsubsection{SUSTENTABILIDADE NAS UNIVERSIDADES - DESAFIOS PARA AS INSTITUIÇÕES}

A promoção da sustentabilidade nas instituições oferece muitas possibilidades. Proporcionar oportunidades de aprendizagem no currículo das instituições de ensino superior se torna relevante para promover mudanças. Isso demanda que essas instituições invistam na formação de seus funcionários, 
agregando ferramentas de ensino e aprendizagem, revisando cursos existentes e desenvolvendo novos cursos, todos com foco na sustentabilidade, em um processo que incorpore os princípios da sustentabilidade na instituição como um todo (TILBURY, 2004; CORCORAN; WALS, 2004).

No entanto, a promoção da sustentabilidade na educação superior tem se confrontado com alguns desafios. Um primeiro mostra que a experiência internacional revela que a maioria das instituições de ensino superior tem obtido um enfoque fragmentado para a sustentabilidade, com foco em iniciativas de "esverdeamento" do campus ou de adicionar conteúdo a uma parte específica do currículo (SPRINGETT; KEARINS, 2005; STARIK; RANDS, 2009; RAUFFLET; DUPRÉ; BLANCHARD, 2009), e que as universidades têm permanecido em grande parte como organizações "que conhecem", em vez de organizações que "aprendem" (TILBURY, 2004).

Senge (2008) define as "organizações que aprendem" como aquelas que aprendem a desenvolver novas habilidades e capacidades, que levam a novas percepções e sensibilidades, que, por sua vez, revolucionam crenças e opiniões (ciclo de aprendizado profundo).

O segundo desafio se relaciona com a organização por disciplinas nas universidades. A promoção da interdisciplinariedade como uma condição para a educação da sustentabilidade tem encontrado muita resistência nas instituições, por razões tanto administrativas como pela relutância de os docentes formados em uma visão disciplinar se engajarem com a interdisciplinariedade e com abordagens mais práticas (experiential learning ${ }^{4}$ ) relacionadas com a educação para a sustentabilidade.

O terceiro desafio está relacionado com o processo organizacional dentro das instituições. Vários autores têm se debruçado sobre essa questão, desde a década de I990, e mostram a necessidade de uma mudança profunda e duradoura. O ensino superior deve abordar a sustentabilidade de forma mais sistêmica, que envolva toda a instituição nas mudanças em andamento, em colaboração com estratégias de aprendizagem (FIEN, I993; HUCKLE; STERLING, I996; STERLING, 2004; TILBURY, 2004). O desafio é ampliar as visões de colaboração e, como Senge (2008) aponta, incluir a participação de toda a gama de stakeholders, o compromisso com iniciativas estratégicas de longo prazo, a adaptabilidade, a importância contextual e a aprendizagem organizacional.

Experiential learning é o processo de construção de significado diretamente da experiência prática. De acordo com Aristóteles: "Para aquilo que temos que aprender antes que possamos fazê-lo, aprendemos fazendo". David A. Kolb ajudou a popularizar a ideia de experiential learning fundamentando-se no trabalho de John Deway, Kurt Lewin e Jean Piaget. Seu trabalho sobre o conceito de experiential learning contribuiu para a expansão da filosofia de experiential education. 


\section{COMPETÊNCIAS PARA O DESENVOLVIMENTO SUSTENTÁVEL}

Para vencer tais desafios relacionados ao significado e à forma de educação para a sustentabilidade em escolas de Administração, a reflexão aqui se apoia em pesquisa conduzida sobre as competências para o desenvolvimento sustentável (TILBURY; WORTMAN, 2004). Fundamentando-se em um processo de pesquisa em profundidade, Tilbury e Wortman (2004) identificaram cinco competências para o desenvolvimento sustentável. Elas são consideradas importantes para que indivíduos, grupos, organizações e sociedades atinjam o que poderia ser considerado um mundo sustentável. As cinco competências são listadas e explicadas no Quadro I.

\section{QUADRO I}

\section{CINCO COMPETÊNCIAS PARA O EDUCATION FOR SUSTAINABLE DEVELOPMENT (ESD) E EXEMPLOS}

\begin{tabular}{ll}
\hline COMPETENCIA & DESCRIÇÃo \\
\hline Perspectivas de futuro & $\begin{array}{l}\text { Ser capaz de imaginar um mundo melhor. } \\
\text { A premissa é que, se sabemos aonde queremos } \\
\text { chegar, seremos mais capazes de trabalhar } \\
\text { para chegarmos lá. }\end{array}$ \\
\hline Pensamento crítico e reflexão & $\begin{array}{l}\text { Aprender a questionar nossos atuais sistemas de } \\
\text { crenças e reconhecer as hipóteses subjacentes } \\
\text { em nosso conhecimento, nossas perspectivas e nossas } \\
\text { opiniões. Habilidades de pensamento crítico podem } \\
\text { ajudar as pessoas a aprender a examinar as } \\
\text { estruturas econômicas, ambientais, sociais e culturais } \\
\text { no contexto do desenvolvimento sustentável. }\end{array}$ \\
\hline Pensamento sistêmico & $\begin{array}{l}\text { Reconhecer as complexidades, ao procurar } \\
\text { por ligações e sinergias, tentando encontrar soluções } \\
\text { para os problemas. }\end{array}$ \\
\hline Construção de parcerias & Promover o diálogo e a negociação, aprender a trabalhar \\
decisão & em equipe. \\
\hline
\end{tabular}


O relatório de 2004 da International Union for the Conservation of Nature (IUCN) (TILBURY; WORTMAN, 2004), denominado Education for sustainable development (ESD), apresenta uma proposta na qual se afirma que, para transformar ESD em uma ferramenta efetiva de engajamento de pessoas para negociar um futuro sustentável e tomar decisões assim, como de agir em nome delas, torna-se necessário que inicialmente se enfrente como a educação pensa a sustentabilidade e a própria educação.

Para tanto, coloca-se o desafio de abordar cinco aptidões essenciais para avançar na direção de um futuro mais sustentável (RAUFFLET; DUPRÉ; BLANCHARD, 2009): I. uma visão de futuro; 2. o desenvolvimento do pensamento crítico e reflexivo; 3 . um pensamento sistêmico que insere complexidades e busca relações e sinergias em busca de soluções para problemas; 4. construção de parcerias, promovendo diálogo e negociação, aprendendo a trabalhar em conjunto; 5. fortalecimento de processos decisórios participativos, por meio do empoderamento de um amplo conjunto de pessoas.

Duas são as vantagens desse quadro de competências relacionadas, pois, em primeiro lugar, permite ir além da visão fundamentada no conhecimento sobre sustentabilidade, o que atrairia apenas instrutores para que incluíssem conteúdos específicos em seus cursos, com conteúdos sobre sustentabilidade que tendem a mudar rapidamente, na medida em que um novo entendimento da ciência da sustentabilidade surge a cada dia; em segundo, essas cinco competências possibilitam a formulação de um curso que vá além dos tradicionais silos, pois elas representam dimensões interdisciplinares e não orgânicas que se mostram inter-relacionadas.

\section{PARTE 3: IMPLICAÇÕES PARA AS ESCOLAS DE ADMINIISTRAÇÃO}

O ensino da Administração tem sido criticado, sob diversos aspectos, na última década, por ser não relevante e desvinculado da prática (MINTZBERG, 2004) e por treinar pessoas com base em uma perspectiva econômica, à custa de abordagens mais integradas (GHOSHAL, 2005).

Uma tradição sólida e a especificidade do ensino da Administração afetam o desenvolvimento de habilidades na formação dos futuros administradores. Conteúdos associados ao desenvolvimento sustentável, que, em geral, têm sido pouco enfatizados nos programas acadêmicos tradicionais, passam a fazer parte com mais frequência. 
Nesta seção do texto, identificam-se principalmente as pressões externas que possibilitam avanços do tema sustentabilidade nos cursos de Administração, a sustentabilidade que está sendo transmitida, assim como as limitações desses avanços.

\subsection{COMO A SUSTENTABILIDADE É APRESENTADA NAS ESCOLAS DE ADMINISTRAÇÃO DE EMPRESAS?}

O ensino de Administração enfatiza significativamente a prática, portanto a adoção de novas teorias nos cursos de Administração surge, na maioria das vezes, de uma necessidade prática ou de uma demanda das empresas, ou seja, de fora para dentro.

Na última década, o surgimento de uma miríade de teorias relacionadas à prática de Corporate Social Responsibility (CSR) e ao Desenvolvimento Sustentável na Administração não deixa de espelhar essa tradição de internalização da área, e, hoje, tal resposta se deve à pressão de governos e sociedades para que as empresas sejam responsabilizadas ou se responsabilizem por seu impacto ambiental, econômico e social.

O conceito de sustentabilidade tem sido difundido nas escolas de Administração de duas maneiras: a primeira, por meio da introdução de novos cursos, tais como CSR ou Sustainability, nos programas. Corporate Social Responsibility (CSR) ou Corporate Sustainability é uma forma de condução dos negócios que cria valor de longo prazo para os acionistas, por meio da adoção de práticas que se aproveitam de oportunidades e gerenciam riscos derivados do desenvolvimento econômico, ambiental e social (KROSS, 2009; PORTER; KRAMER, 2006; ZADEK, 2004). A premissa de atuação com base na CSR comumente representa uma resposta das corporações às políticas e às ações relacionadas aos desafios da sustentabilidade.

O pensamento dominante da Administração, em suas definições do conceito de sustentabilidade nas organizações, fundamentalmente aborda a questão social e os desafios postos pelas questões ambientais como oportunidades de negócio, seja como uma forma de reduzir custos (PORTER; VAN DEN LINDE, I995), seja como uma forma de posicionamento diferenciado de produtos e serviços.

No entanto, tanto os cursos de Administração quanto as empresas que buscam estar na vanguarda da aplicação das teorias relacionadas ao desenvolvimento sustentável ainda tratam a questão como uma resposta à cobrança da sociedade e à necessidade de se manterem uma imagem e uma reputação de organização socialmente responsável e, principalmente, como uma resposta a exigências legais e de licença para operar. Ao menos na prática, a responsabilidade social das empresas se mostra ainda apenas reativa. 
Em teoria, o princípio da sustentabilidade, nas escolas de Administração, é amplamente fundamentado no tão conhecido triple bottom line da performance econômica, social e ambiental. Mas mesmo a teoria ensinada nos cursos de Administração ainda é deficiente em ferramentas que podem ajudar os tomadores de decisões nas empresas a escolher entre um projeto social e outro. Ou seja, princípios de sustentabilidade e responsabilidade social ainda são ensinados e vistos como uma obrigação moral e, na maioria dos casos, também legal pelos cursos de Administração e pelas empresas, como uma necessidade de olhar para o futuro, a fim de não ser surpreendido por demandas da sociedade quanto a questões que o administrador não havia se dado conta de que seriam parte da responsabilidade das empresas.

Tal como o conceito e as práticas de CSR não fazem parte das decisões estratégicas da maioria das empresas, também ainda no ensino da Administração tais conceitos se encontram dissociados do centro da teoria geral da Administração e, em muitos casos, até mesmo em conflito. Existe ainda um longo caminho a percorrer, antes que a sustentabilidade se torne o esteio de um programa para o ensino da Administração (STARIK; RANDS, 2009). Até o momento, a maior parte dos avanços foi alcançada com a introdução de cursos ad hoc relacionados com a sustentabilidade no currículo existente, e sua introdução parcial não ocorreu sem problemas.

Giaccalone e Thompson (2006b) destacaram a diferença entre premissas implícitas e explícitas na ética de negócios nos programas de gestão. Enquanto um currículo "implícito" se refere à ideologia e às premissas subjacentes dos cursos específicos e disciplinas, um currículo "explícito" se refere a tentativas formais e deliberadas de introduzir o tema da sustentabilidade nos diálogos e na agenda de interesses.

A inclusão de um módulo ou de um curso sobre desenvolvimento sustentável em um programa dominado por um paradigma econômico é imediatista e provavelmente fracassará no sentido de direcionar mudanças profundas em um pensamento focado apenas na Administração e na demanda por sustentabilidade (GHOSHAL, 2005).

A segunda forma tem consistido na promoção da sustentabilidade como uma oportunidade em determinada disciplina, o que representa a frequentemente criticada forma de organização de silos de conhecimento nas escolas de Administração (MINTZBERG, 2004).

O exemplo da introdução da ecoeficiência no campo de produção e logística ilustra tal foco em uma única disciplina. O conceito da ecoeficiência ${ }^{5}$ se tornou 
moda no campo da produção e logística nos últimos quinze anos. Ecoefiência se tornou uma meta para as empresas em todo o mundo, e muitas chegaram a concretizar significativas reduções de custos em decorrência de tais inovações.

Embora tal desenvolvimento possa ser considerado louvável, a simples adoção de inovações ecoeficientes pode até mesmo piorar o estresse causado ao meio ambiente no futuro (SENGE; CARSTEDT, 2002). Tais inovações reduzem a produção de resíduos no processo produtivo, mas não alteram o número de produtos manufaturados ou alteram a quantidade de resíduos gerados por seu uso e descarte; de fato, muitas empresas investem em ecoeficiência para aumentar crescimento e lucro. Adicionalmente, não há garantias de que o crescimento econômico gerado pela ecoeficiência traduzir-se-á em investimentos também ecoeficientes e que não será reinvestido em indústrias ecoineficientes.

Até um vasto sistema industrial ecoeficiente pode, caso venha a crescer muito, gerar mais resíduos e destruir mais habitats e espécies do que uma menor e menos eficiente economia. Logo, para a sustentabilidade de uma atividade econômica, a ciência administrativa deve desenvolver uma nova abordagem sistemática que permita às empresas reduzir a utilização total de insumos no processo produtivo e o total de resíduos acumulados em toda a cadeia distributiva e o ciclo de vida dos produtos.

Tal foco na sustentabilidade em uma única disciplina tem o potencial de melhorar a eficiência. No entanto, tal estratégia provavelmente falhará em seu questionamento e no redesenho de todo o processo.

Raufflet (2006) propôs três formas de gestão ambiental das empresas com base em três paradigmas. O primeiro, incremental, envolve as operações de negócios e tem um comportamento essencialmente reativo, no qual as empresas reduzem seu impacto direto sobre a biosfera. A segunda é adaptativa e procura desenhar a produção e os sistemas organizacionais com base na visão da dinâmica ecossistêmica que inclui. Essa segunda forma de gestão ambiental corporativa tenta reorganizar as atividades corporativas em termos de fluxos e processos de energia integrados e interdependentes com os de outras empresas. A terceira forma de gestão ambiental é radical e se fundamenta na premissa de que as formas de produção e consumo atuais têm levado a uma degradação ambiental do planeta, impondo um padrão insustentável de consumo dos recursos naturais, poluindo o ecossistema acima dos limites controláveis e estimulando elevados padrões de consumo por uma parcela muito restrita da população, acentuando, consequentemente, as desigualdades no acesso aos recursos naturais.

O que se pode constatar é que no ensino da Administação prevalece o primeiro e o segundo paradigmas.

Pode-se argumentar que a maior parte do material e dos módulos relacionados à sustentabilidade nas escolas de Administração se refere a paradigmas 
incrementais e adaptativos. O foco está no treinamento de futuros administradores para que possam trabalhar em direção a um aumento da eficiência e da geração de inovações incrementais. Muito pouco tem sido feito para instigar estudantes a repensar e a desafiar o sistema mais amplo de produção, em seu contexto ecológico-social. Em outras palavras, sustentabilidade nas escolas de Administação apenas considera marginalmente o fato de vivermos em um planeta com recursos finitos.

\subsubsection{OBSTÁCULOS AOS AVANÇOS DA SUSTENTABILIDADE NO CURRÍCULO DAS ESCOLAS DE ADMINISTRAÇÃO DE EMPRESAS}

Com base em uma revisão da literatura, identificaram-se quatro limitações para o avanço da sustentabilidade no currículo das escolas de Administração. A primeira limitação está relacionada com os obstáculos de base disciplinar, dentro das disciplinas. A divisão de conhecimento se transforma em um obstáculo, quando poderia contribuir para a integração da sustentabilidade no currículo.

Benn e Martin (20I0) apresentam uma revisão da literatura, na qual destacam barreiras, incentivos e metodologias que permeiam a integração do tema da sustentabilidade nas escolas de Administração. Entre os aspectos mais relevantes, verifica-se que, no geral, existe um crescimento da inserção do enfoque da sustentabilidade em muitas instituições, entretanto a inclusão/inserção do tema nas disciplinas específicas, assim como o ensino em torno de conceitos associados, ocorre notadamente a partir de iniciativas individuais de docentes, mas pouco articuladas com o todo institucional.

O segundo obstáculo está relacionado com as abordagens de ensino. O método de estudo de caso representa a abordagem de ensino dominante nas escolas de Administração que promovem um ensino no qual os estudantes são estimulados a tomar decisões como indivíduos focados predominantemente em uma visão de maximização de curto prazo de um pequeno grupo, com base em uma análise de custo-benefício.

Essa abordagem se contrapõe à visão educacional mais inclusiva e participativa, que caracteriza o desenvolvimento sustentável. Para Benn e Martin (20I0), uma das questões que mais desafiam as universidades e, no caso, as escolas de Administração é como as instituições podem modificar sua dinâmica de aprendizagem organizacional, visando enfatizar uma visão participativa e abrangente que inclua a universidade e a sociedade no tema da sustentabilidade, principalmente os enfoques participativos, em uma visão mais integradora e abrangente. 
O terceiro obstáculo se relaciona com o valor da sustentabilidade e o desafio de sua mensuração. Segundo Bradfield (2009), a lentidão das escolas de Administração em modificar seus currículos decorre do fato de que ainda é muito incipiente a aplicação da sustentabilidade como conceito ou valor (VEIGA, 20IO).

Observa-se que a inovação está centrada nas abordagens técnicas e em questões associadas às características dos produtos (ciclo de vida), praticamente desconsiderando aspectos menos tangíveis relacionados com a sustentabilidade.

Para Starkey e Tempest (2008, p. 387), ainda são poucas as iniciativas que focam os temas da complexidade e do risco como estruturantes da formação (BECK, I992). Vários novos drivers têm provocado algumas transformações cujos alcances ainda são pouco mensuráveis, mas que colocam novas perspectivas no posicionamento das instituições.

Provavelmente a necessidade de entender as complexidades colocadas pela perspectiva da mudança climática promova uma agenda de pesquisa e ensino que poderá romper com uma lógica convencional de pensar disciplinarmente, de forma a introduzir a articulação interdisciplinar como foco de articulação conceitual.

O quarto desafio está relacionado com a integração, na medida em que se coloca a necessidade de apresentar e usar um paradigma de complexidade em um contexto que estimule o pensamento linear.

Nesse sentido, esse desafio propõe a metodologia da resolução de problemas no sentido de estimular complexas abordagens e aquisição de habilidades em uma agenda de ensino orientada por valores instrumentalistas.

\section{PARTE 4: COMO INTEGRAR SUSTENTABILIDADE NO ENSINO DA ADMINISTRAÇÃO?}

Propõe-se o desenvolvimento de princípios básicos, com base no marco conceitual de Tilbury e Wortman (2008), como uma forma de integrar a sustentabilidade nos cursos de Administração.

O primeiro princípio está relacionado com o pensamento sistêmico: o ensino de conceitos de sustentabilidade deve fazer parte do currículo obrigatório, na formação de um administrador, e também deve ser parte de atividades extracurriculares, se a instituição de ensino busca oferecer a seus alunos uma visão holística e estratégica sobre sustentabilidade. Essa visão estratégica e holística é a forma como qualquer tópico, não apenas questões permeadas pela sustentabilidade, é abordado pelos tomadores de decisão nas empresas. 
O segundo está relacionado com a interdisciplinariedade. A ciência da Administração tem em sua origem uma construção interdisciplinar, aplicada aos desafios da prática de gestão, que faz com que suas diferentes áreas se complementem e coexistam em consonância. Portanto, as escolas de pensamento da Administração devem buscar caminhos para inserir as questões de sustentabilidade de forma que suas discussões façam parte do desenvolvimento dessa própria ciência, e não mais sejam parte de um movimento de diferenciação usado por algumas instituições de ensino que, muitas vezes, entram em conflito com suas diversas áreas.

Se o conhecimento gerado em finanças deve ser coerente com o que se aprende em outras áreas, também se deve buscar a coerência com as questões postas pelos desafios da sustentabilidade.

O terceiro princípio está relacionado com os três pilares do ensino de desenvolvimento sustentável para a tomada de decisões. De acordo com a Unesco (2005), a educação ambiental deve considerar as três dimensões da sustentabilidade - social, ambiental e econômica -, pois isso permite que as pessoas desenvolvam as habilidades necessárias, o conhecimento e as perspectivas para tomarem decisões que melhorarão a qualidade de vida em todos os níveis. E isso só pode ocorrer se o ensino da Administração, em todas as suas áreas, estiver de acordo, simultaneamente, com tais dimensões do desenvolvimento sustentável.

Duas condições precisam ser atendidas nos esforços para a promoção do desenvolvimento sustentável no currículo das escolas de Administração. Até o momento, muitas iniciativas surgiram desde os anos I990, e os desafios para a próxima década consistem em sua capilarização nas instituições.

A primeira condição para que que os desafios da sustentabilidade consigam integrar eficientemente o ensino da administração é planejar como a sustentabilidade será implantada: por meio de estruturas já existentes ou com a criação de novas estruturas.

A segunda condição é relativa ao status de sustentabilidade em um contexto predominantemente disciplinar dos cursos de Administração. A sustentabilidade em geral é percebida como "branda", entre um conjunto de temas. Também em contraposição, nas disciplinas clássicas, como Economia, Finanças, Gestão de Recursos Humanos, o desenvolvimento sustentável carece de uma sólida base epistemológica. No entanto, o que ainda se vê na maioria das instituições de ensino de Administração é o pouco prestígio dado à sustentabilidade.

Maior comprometimento com a inserção das questões de sustentabilidade no currículo do curso de Administração gera, sem dúvida, maior demanda por educadores e recursos, mas, no modelo atual, grande parte dos educadores pre- 
fere ensinar as disciplinas tradicionais do currículo de Administração, visto que tal opção possui maiores chances de promoção, segurança no emprego e outros benefícios e recompensas.

\section{REFLEXÕES FINAIS: NOVOS CAMINHOS PARA OS CURSOS DE ADMINISTRAÇÃO}

Neste artigo, destacaram-se o contexto global da sociedade de risco e o contexto no qual a sustentabilidade, associada com a educação, tem sido promovida nos cursos de ensino superior e, especificamente, nos cursos de Administração. Mapearam-se os resultados e foram identificados os desafios para o avanço da sustentabilidade no curso de Administração.

Esta última seção propõe quatro caminhos para o fortalecimento das ideias de sustentabilidade nos cursos de Administração na próxima década.

\subsection{DA APRENDIZAGEM INDIVIDUAL À APRENDIZAGEM COLETIVA - CONSTRUINDO COMUNIDADES DE APRENDIZAGEM}

A expressão comunidades de aprendizagem, tal qual como utilizada neste artigo, refere-se a um grupo de pessoas que divide as mesmas emoções, os mesmos valores e as mesmas crenças, e está ativamente engajado em aprender em conjunto e uns com os outros, por habituação.

Tais comunidades se tornaram um modelo para uma abordagem interdisciplinar fundamentada no grupo para a educação superior. Essa abordagem pode basear-se em um desenho de educação avançada ou pedagógica. Isso configura uma situação na qual a questão climática passa a ser vista como um tema que pode romper com as barreiras disciplinares, criando um potencial de maior diálogo com as novas fronteiras de conhecimento.

As formas tradicionais de ensino, fundamentadas, no geral, em solução de problemas administrativos, começam a ser cada vez mais questionadas (BENN; MARTIN, 20IO). Isso introduz um tema que ainda é pouco disseminado no Brasil: as possibilidades de ampliar as lógicas de aprendizagem por meio da ênfase em metodologias, como é o caso das "comunidades de práticas".

A comunicação entre as pessoas pode potencializar interações que tragam avanços substanciais na produção de novos repertórios e práticas de mobilização social para a sustentabilidade (GLASSER, 2007; STERLING, 2004; WENGER, I998). Para Wenger (I998, p. 229): 
As comunidades de prática são grupos de pessoas que compartilham uma preocupação ou paixão por algo que fazem e aprendem como fazê-lo melhor na medida em que interagem com regularidade. Os participantes da comunidade interagem em ações e discussões, apoiam-se mutuamente, trocam informações, aprendem juntos. Desenvolve-se um repertório compartilhado de recursos: experiências, histórias, ferramentas, modos de lidar com problemas recorrentes - em suma, uma prática compartilhada.

Wenger (I998) destaca o aprendizado como um fenômeno que reflete a natureza social do homem, no contexto de suas experiências de participação no mundo, e alerta para a lacuna do sistema educacional que, frequentemente, articula o ensino como se o aprendizado fosse um processo individual e desconectado das demais experiências da vida. Aponta, assim, que é na perspectiva da valorização do caráter social do aprendizado e dos aspectos colaborativos envolvidos que as comunidades de prática podem ser analisadas como promotoras de aprendizagem, enfatizando que grande parte do dia a dia do indivíduo envolve essas comunidades.

Conforme Glasser (2007), a distância entre a consciência e as práticas é decorrente da cultura da qualidade de vida centrada na lógica do bem-estar do indivíduo e do estímulo ao consumo, o que favorece a desigual distribuição da renda e a falta de responsabilidade com a vida do planeta.

Para esse autor, a transição para uma "sustentabilidade ecocultural” demanda, entre outras coisas, a valorização e o cuidado com a diversidade cultural e biológica; a promoção de formas democráticas de governança; e o favorecimento de economias de base local, articulada com estilos de vida que respeitem os limites da natureza. Além disso, destaca que os processos de aquisição de práticas mais sustentáveis exigem a familiaridade com elas, que nem sempre são conhecidas ou lembradas, e sua percepção como mais convenientes do que as práticas insustentáveis.

Instaura-se, assim, a importância de reconhecer a existência de comunidades de prática orientadas para a promoção da sustentabilidade e de fortalecê-las. Trata-se de comunidades que não podem ser impostas, mas sim imaginadas, identificadas, estimuladas, cultivadas e valorizadas.

Nesse sentido, Wenger (1998) recomenda que se pense em formas criativas de engajar os estudantes em práticas significativas, providenciando meios para que possam criar comunidades de prática, ingressar nas que já existem e ampliar sua interação naquelas em que já participam, como ação estratégica para alargar o potencial de aprendizagem e de formação da identidade dos estudantes. 


\subsection{EDUCAÇÃO PARA A SUSTENTABILIDADE - NA DIREÇÃO DA APRENDIZAGEM SOCIAL}

O que se observa é que a educação para a sustentabilidade ainda se situa em um contexto em que prevalece uma lógica prescritiva, e isso limita, segundo Sterling (2004) e Tilbury e Wortman (2004), uma visão que enfatize uma abordagem que transcenda fronteiras disciplinares. Para tanto, coloca-se a necessidade de abordagens sistêmicas de sustentabilidade que reforcem dimensões participativas e o fortalecimento de valores em diálogo com perspectivas adaptativas (PORTER; CORDOBA, 2009), enfatizando abordagens que transcendam o locus acadêmico. Assim, a educação para a sustentabilidade demanda a incorporação das premissas de Wenger (I998), que enfatizam os princípios de aprendizagem social - comunidades de prática -, possibilitando uma aprendizagem pautada por interesses comuns, que promovam as dimensões da sustentabilidade e suas interconexões por meio de diversos meios.

A aprendizagem que enfatiza as fronteiras potencializa inovação no conhecimento, rupturas epistemólogicas, ampliando espaços interativos e podendo gerar novos conhecimentos, assim como o compartilhamento mais articulado de ideias e capacidades (WENGER, 2000).

\subsection{INTEGRACAO DO CONHECIMENTO: A SUSTENTABILIDADE COMO JANELA DE OPORTUNIDADE NOS CURSOS DE ADMINISTRAÇÃO}

Da leitura de alguns textos (ALCARAZ; THIRUVATTAL, 20IO; OLSSON; FOLKE; BERKES, 2004; UNESCO, 2005), o que diversos pesquisadores e especialistas mostram é que haverá uma redefinição do quadro de ensino/ aprendizagem, principalmente a presença da dimensão sustentabilidade como fator inovador.

O desafio de ser inovador se apresenta no sentido de que a inovação para a sustentabilidade representa o que tem sido recentemente denominado como “janela de oportunidade” para as escolas de Administração na formação de líderes.

Dado o nível de competição entre as empresas, um dos maiores desafios é inovar na formação. E a pergunta que se coloca é: “Como?". Considerando o quadro de profissionais e sua diversidade, além do fato de que, para a maioria, a sustentabilidade não é tema central da agenda de conhecimento, e, além disso, ainda há uma visão estereotipada sobre sustentabilidade, torna-se necessário avançar na direção de alguns quadros conceituais que promovam mudança e adaptação para os novos tempos. 
Dois movimentos nos desafiam: de um lado, um posicionamento institucional favorável às mudanças, não pautado por visões casuísticas ou oportunistas, mas como um componente estruturante da gestão acadêmica; de outro, a importância de que os docentes comprometidos e engajados com a visão de educação para a sustentabilidade possam desenvolver e, se possível, multiplicar sua visão multidisciplinar.

\subsection{ALÉM DO TREINAMENTO GERENCIAL: EDUCANDO INDIVÍDUOS RESPONSÁVEIS E COMPROMETIDOS COM A SUSTENTABILIDADE}

Por último, mas não menos importante, a sustentabilidade também representa um convite para que se possa repensar o papel do ensino da Administração em relação à educação de estudantes e futuros administradores.

O propósito dos três caminhos previamente identificados (construção de uma comunidade de aprendizagem, aprendizagem social e integração do conhecimento) é repensar e potencialmente melhorar aquilo que é a missão central do ensino da Administração: a educação de gestores.

Até o momento, o ensino da Administração tem enfatizado o papel dos gestores em detrimento de outros papéis e responsabilidades que indivíduos e grupos possuem na sociedade. Educar indivíduos além de seus papéis professionais e gerenciais, dados os desafios que a humanidade vem enfrentando e enfrentará, representa uma carga de responsabilidade para o ensino da Administração no século XXI e um convite também para se reinventar.

\section{EDUCATION FOR SUSTAINABILITY IN MANAGEMENT COURSES: REFLECTION ON PARADIGMS AND PRACTICES}

\section{ABSTRACT}

There has been a multiplication of sustainability related modules, courses, programs over the last decade in Higher Education in general, as well as more specifically in Business Education. The purpose of this paper is to provide a critique/evaluation of these efforts and to map avenues for the design of modules, courses and programs through a reflection on education paradigms and practices. This paper' goal is to contribute to the debate about the role of higher education, notably the role of administration and management courses, as it is observed a 
significant increase in the number of institutions of higher education that have an effective motivation to educate students with knowledge and skills needed to put sustainability at the core of its future management activities. It presents a reflection on the main strands of thought, about the main debates, it identifies ambiguities and contradictions in the debate and presents the possibilities of integrating the theme of sustainability in business administration courses. The authors bring up the issue of sustainability in its dialogue with companies and academia, the implications of the emergence of risk society to higher education and business schools. It also discusses the current schools of thought in economics and ecology proposals for education within paradigms that address complexity, the inclusion of sustainability in higher education and management education. The paper is divided into four sections: the first maps the risk society and the recent economic thought, outlines the reasons/assumptions of society and education paradigms. The second part presents a survey on promotion/dissemination of sustainability in higher education, highlighting the context, outcomes and challenges. The third focuses on sustainability in management education and its major challenges. The last section proposes three ways to integrate the theme of sustainability in management education, skills for sustainable development and finally, the implications, obstacles and opportunities for integrating sustainability in management education. The article dialogues with the latest international literature and seeks to draw readers' attention on key conceptual challenges to educate individuals responsible and committed to sustainability.

\section{KEYWORDS}

Sustainability; Higher education; Business education; Education paradigms and praxis; Risk society.

\section{EDUCACIÓN PARA LA SOSTENIBILIDAD EN CURSOS DE GESTIÓN: REFLEXIÓN SOBRE PARADIGMAS Y}

\section{RESUMEN}

En la última década se multiplicaron los módulos, cursos y programas relacionados con la sostenibilidad en la enseñanza superior de forma general, y especialmente en la enseñanza de gestión. El objetivo de este artículo es de presentar 
reflexión y evaluación de las iniciativas y mapear caminos posibles para la construcción de módulos, cursos y programas a través de análisis sobre los paradigmas educativos y sus prácticas. Trata-se de contribuir al debate sobre el papel de los cursos de administración y gestión, en la medida que se observa importante aumento del número de instituciones de enseñanza superior que presentan una efectiva motivación para formar estudiantes con conocimiento y habilidades necesarias para colocar el tema de la sostenibilidad en el centro de sus futuras actividades de gestión. Se presenta una reflexión sobre las principales corrientes de pensamiento y los debates actuales, se identifican ambigüedades y contradicciones y las posibilidades de integración del tema de la sostenibilidad en los cursos. Los autores destacan el tema de la sostenibilidad en las empresas y su dialogo con el campo académico, los alcances de la emergencia del paradigma de la sociedad de riesgo para la educación superior y las escuelas de administración. También abordan las corrientes de pensamiento de la economía y la ecología, y propuestas para la educación dentro de paradigmas que traten de la complejidad y la inserción de la sostenibilidad en los cursos. El artículo está estructurado en cuatro secciones: la primera mapea la sociedad de riesgo y el pensamiento económico reciente, y destaca los fundamentos así como los paradigmas de la educación. La segunda presenta un análisis sobre promoción/ difusión de la sostenibilidad en la educación superior, destacando contexto, resultados y desafíos. La tercera enfoca la sostenibilidad en la enseñanza de administración y sus principales desafíos. La última sección propone tres caminos para integrar el tema de la sostenibilidad, las capacidades para el desarrollo sostenible y finalmente las implicaciones, obstáculos y posibilidades de integrar de forma efectiva la sostenibilidad en los cursos. El artículo dialoga con la literatura internacional más actual y busca interesar a los lectores sobre los principales desafíos conceptuales para educar individuos responsables y comprometidos con la sostenibilidad.

\section{PALABRAS CLAVE}

Sostenibilidad; Enseñanza superior; Enseñanza de Administración; Paradigmas educativos; Sociedad de riesgo.

\section{REFERÊNCIAS}

ALCARAZ, J.; THIRUVATTAL, E. An interview with Manuel Escudero: the united nations' principles for responsible management education: a global call for sustainability. Academy of Management Learning \& Education, Birmingham, v. 9, n. 3, p. 542-550, Sept. 2010. 
ARISTÓTELES. Ética à Nicômaco. São Paulo: Martin Claret, 200 I. ASSOCIATION OF UNIVERSITY LEADER FOR A SUSTAINABLE FUTURE. The Talloires Declaration - Io point action plan. Taillones: Association of University Leader for a Sustainable Future, I990.

BECK, U. Risk society: towards a new modernity. London: Sage, I992.

BECK, U.; GRANDE, E. Varieties of second modernity: extra-European and European experiences and perspectives. British Journal of Sociology, London, v. 6I, n. 3, p. 406-638, Sept. 2010.

BENN, S.; MARTIN, A. Learning and change for sustainability reconsidered: a role for boundary objects. Academy of Management Learning \& Education, Sydney, v. 9, n. 3, p. 397-4I2, Sept. 2010. BRADFIELD, S. The value of sustainability education. Journal of Management Education, London, v. 33, n. 3, p. 372-375, Sept. 2009.

BUSINESS STRATEGY AND THE ENVIRONMENT CONFERENCE. Devonshire Hall: University of Leeds, 2005 .

CALVO, R. S.; BENAYAS, J.; GUTIERREZ, P. J. Learning in sustainable environments: the greening of higher education. In: TILBURY, D. et al. Education and sustainability: responding to the global challenge. Cambridge: IUCN Publications Services Unit, 2002.

CORCORAN, P. B.; WALS, A. E. J. Higher education and the challenge of sustainability: problematics, promise, and practice. Dordrecht: Kluwer Academic Publishers, 2004.

DALY, H. Steady-state economic: the economics of biophysical equilibrium and moral growth. San Francisco: W. H. Freeman, I977.

FIEN, J. Environmental education: a pathway to sustainability. Geelong: Deakin University Press, I993.

GHOSHAL, S. Bad management theories are destroying good management practices. Academy of Management Learning and Education, London, v. 4, n. I, p. 75-91, Mar. 2005.

GIACCALONE, R. A.; THOMPSON, K. R. Special issue on ethics and social responsibility. Academy of Management Learning and Education, Austin, v. I6, n. 4, p. 26I-265, Mar. 2006 a.

Business ethics and social responsibility education: shifting the worldview. Academy of Management Learning and Education, Austin, v. I6, n. 4, p. 266-277, Mar. $2006 \mathrm{~b}$.

GLASSER, H. Minding the gap: the role os social learning in linking our stated desire for a more sustainable world to our everyday actions and policies. In: WALS, A. E. J. (Ed.). Social learning towards a sustainable world: principles, perspectives, and praxis. Wageningen: . Wageningen Academic, 2007. p. 35-6I.

HARDING, G. The tragedy of the commons. Logan: Association for the Advancement of Science at Utah State University, I968.

HUCKLE, J.; STERLING, S. Education for sustainability. London: Earthscan, I996.

JACKSON, T. Prosperity without growth? Economics for a finite Planet. London: Earthscan, 2009. KOLB, D. A.; FRY, R. Toward an applied theory of experiential learning. In: COOPER, C. Theories of group process. London: John Wiley, I975.

JACOBI, P. R. Educação ambiental: o desafio da construção de um pensamento crítico, complexo e reflexivo. Revista Educação e Pesquisa FE-USP, São Paulo, v. 3I, n. 2, p. 302-313, maio/ago. 2005. KROSS, K. Profession and purpose. Sheffield: Greenleaf, 2009.

MINTZBERG, H. Managers, not MBAs. San Franciso: Berrett-Koehler, 2004. 
OLSSON, P.; FOLKE, C.; BERKES, F. Adaptive comanagement for building resilience in socialecological systems. Environmental Management, Stockholm, v. 34, n. I, p. 74-90, July 2004.

PORTER, M. E.; KRAMER, R. Strategy \& society: the link between competitive advantage and corporate social responsibility. Harvard Business School Publishing Corporation, Cambridge, v. 84, n. I2, p. 78-92, Dec. 2006.

PORTER, M. E.; VAN DEN LINDE, C. Toward a new conception of the environment - competitivess relationship. Journal of Economic Perspecives, Chicago, v. 9, n. 4, p. 97-II8, Aug. I995.

PORTER, T.; CORDOBA, J. Three views of systems theories and their implications for sustainability education. Journal of Management Education, London, v. 33, n. 3, p. 323-346, Sept. 2009.

RAUFFLET, E. Re-mapping corporate environmental management paradigms. International studies of Management and Organization, Montreal, v. 36, n. 2, p. 54-72, July 2006.

RAUFFLET, E.; DUPRÉ, D.; BLANCHARD, O. Training managers for sustainable development: the lens of three practioners. In: WANKEL, C.; STONER, J. A. F. Management education for global sustainability. Charlotte: Information Age Pub, 2009. p. 377-393.

SENGE, P. M. A quinta disciplina. 24. ed. Rio de Janeiro: Best Seller, 2008.

SENGE, P. M.; CARSTEDT, G. Innovating our way to the next industrial revolution. MIT Sloan Management Review, Cambridge, v. 32, n. I, p. 7-23, Jan. 2002.

SHARP, L. Green campuses: the road from little victories to systemic transformations. International Journal of Sustainability in Higher Education, Richmond, v. 3, n. 2, p. I28-45, May 2002.

SLATER, D. J.; DIXON-FOWLER, H. R. Sustainability - the future of the Planet in the hands of MBAs: an examination of CEO MBA Education and Corporate Environmental Performance. Academy of Management Learning an Education, v. 9, n. 3, Sept. 2010.

SPRINGETT, D.; KEARINS, K. Educating for sustainability: an imperative for action. Business Strategy and the Environment, New York, v. I4, p. I43-I45, May/June 2005.

STARIK, G.; RANDS, M. The short and glorious history of sustainability in North American management education. In: WANKEL, C.; STONER, J. A. F. Management education for global sustainability. Charlotte: Information Age Pub, 2009. p. 377-393.

STARKEY, K.; TEMPEST, S. A clear sense of purpose? The evolving role of the business school. Journal of Management Development, Nottingham, v. 27, n. 4, p. 379-390, Apr. 2008.

STEAD, J. G.; STEAD, W. E. Sustainability comes to management education and research: a story of coevolution. Academy of Management Learning Education (Amle), v. 9, n. 3, p. 488-498, 2010.

STERLING, S. Higher education, sustainability, and the role of systemic learning. In: CORCORAN, P. B.; WALS, A. E. J. Higher education and the challenge of sustainability: problematics, promise, and practice. Dordrecht: Kluwer Academic, 2004. p. 47-70.

THOMAS, I. Sustainability in tertiary curricula: what is stopping it happening? International Journal of Sustainability in Higher Education, Melbourne, v. 5, n. I, p. 33-47, Dec. 2004.

TILBURY, D. Environmental education for sustainability: a force for change in higher education. In: CORCORAN, P. B.; WALS, A. E. J. Higher education and the challenge of sustainability: problematics, promise, and practice. Dordrecht: Kluwer Academic, 2004. p. 97-II2.

TILBURY, D.; WORTMAN, D. Engaging people in sustainability. Gland, Switzerland: ICN, 2004.

Education for sustainability in further and higher education: reflections along the journey. Planning for Higher Education, Ann Arbour, v. 36, n. 4, p. 5-I6, July/Sept. 2008. 
UNESCO. Contributing to a more sustainable future: quality education, life skills and education for sustainable development. 2002. Disponível em: <http://unesdoc.unesco.org/images/ooI4/ oоI4Io/I4Iorge.pdf>. Acesso em: Io jan. 20II.

VEIGA, J. E. Sustentabilidade - A legitimação de um novo valor. São Paulo: Senac, 20 o.

WANKEL, C.; STONER, J. A. F. Innovative approaches to global sustainability. New York: Palgrave Macmillan, 2008.

Management education for global sustainability. Charlotte: Information Age, 2009.

WENGER, E. Communities of practice: learning, meaning, and identity. New York: Cambridge University Press, I998.

Communities of practice and social learning systems. Organization, London, v. 7, n. 2, p. 225-246, Sept. 2000.

ZADEK, S. The path to corporate responsibility. Harvard Business Review, Cambridge, v. 82, n. I2, p. I25-136, Dec. 2004 .

ZELEM, M. C.; BLANCHARD, O.; LECOMTE, D. Education pour le développement durable: de l'école au campus. Paris: L'Harmatlan, 2010. 